\title{
On the Design of Freeway Traffic Safety Information Platform
}

\author{
Nale Zhao ${ }^{1, a}$, Tianran Zhou ${ }^{2, b}$ \\ ${ }^{1}$ Department of Traffic Engineering, Research Institute of Highway, \\ Ministry of Transport, Beijing, China \\ ${ }^{2}$ IP Technology Research Department, Huawei Technologies Corporation, Beijing, China \\ anl.zhao@rioh.cn, bzhoutianran@huawei.com
}

Keywords: Freeway, Information Platform, Geographic Information System (GIS), System Architecture.

\begin{abstract}
With the rapid development of urbanization, freeway with the advantage of high throughput and speed becomes an important part of the urban construction. Complex fators such as normal traffic, special events and environment raise a problem for the freeway traffic safety. In this paper, an informational approach is used to solve this problem. We propose a novel freeway traffic safty information platform and explain the design of key modules. The system and method proposed in this paper will be a reference for the freeway informatization construction in the future.
\end{abstract}

\section{Introduction}

Freeway with the advantage of high throughput and speed impact our life in many ways. The fast growth of traffic volme and freeway mileage raise a great challenge for the safety and management of the freeway. How to improve the capability of safety surveillance and emergency rescue with adverse conditions, for instance bad weather, accident and traffic congestion, are crucial issues need to be considered.

With the rapid development of communication and information science, using the informational approach to improve the freeway traffic safety becomes the first choice for governments. As in England, a national road network management center governing seven regional agencies was established in Birmingham [1].The center receives and processes freeway state data from the seven agencies, and informs road users of conditions on the freeway network by variable message signs, hotlines, traffic radios and the internet. Because of the federalism, the United States of America (USA) does not have a national freeway network management center, but each state has its own monitoring and management system [2]. For the emergency management, the USA has mechanism to coordinates related departments in a district. In the Netherlands, traffic control agencies from five dstricts are managed and coordinated by a national traffic management and information center, which integrates national wide highway management fuctions logically [1]. Policies and coordinated operations are made for emergencies.

In this paper, a freeway traffic safety information platform is proposed. Starting from the analysis of fuctional requirements, the solution and key modules are introduced.

\section{Requirement Analysis}

The freeway traffic safety information platform is a distributed system covering a large area. Functions and features need to be specified before the system design. Here we conclude some basic requirements as follows:

- Traffic in the freeway network can be monitored in real-time. The platform collects monitoring data, accident reports, and traffic volume statistics reports from various road sections in the freeway network. 
- The platform can adjust the traffic dynamically to optimize performance of the entire freeway network. The operation can be accomplished automatically or manually based on the data analysis.

- The platform can generate various report form, and provide functions of querying, printing, and statistic.

- When emergency happens, related freeway sections can be monitored and controlled with higher priority

- Traffic information and warning can be published through multiple channels, e.g. traffic radios, internet, and variable message signs.

- Early-warning and contingency plan can be established for the emergency.

\section{System Architecture}

To build a versatile information platform, the concept of intelligent transportation system is introduced. Information resources are efficiently integrated to help operations of emergency management, traffic operation status analysis, information publication, traffic flow steering and so on.

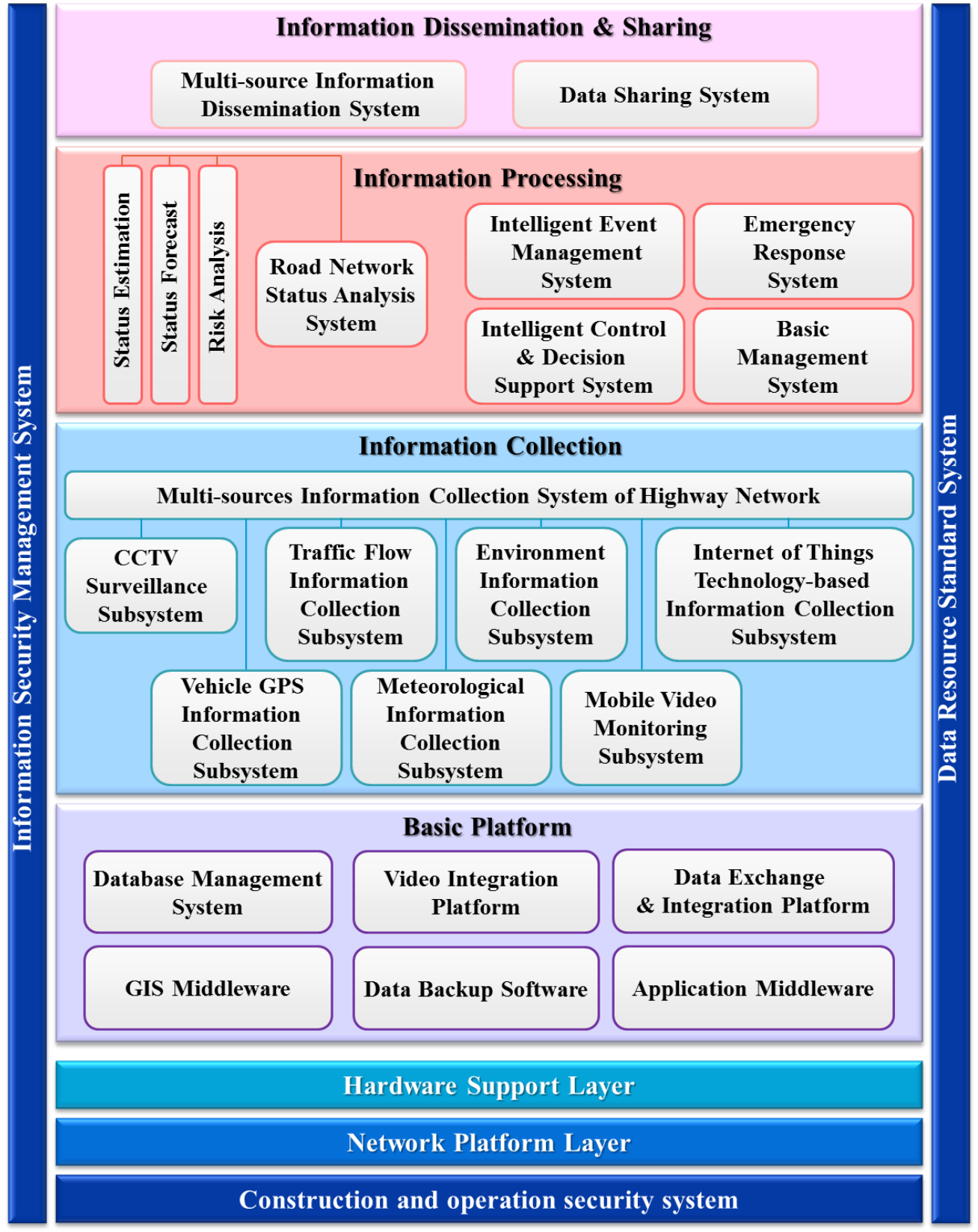

Fig. 1 Architecture of the Freeway Traffic Safety Information Platform 
Fig. 1 shows the architecture of the freeway traffic safety information platform, which can be divided into four layers.

- Basic Platform Layer: provides the software infrastructure for other system layers.

- Information Collection Layer: integrates multiple modern monitoring and information collection approaches.

- Information Processing Layer: generates analysis results and control instructions by processing massive data.

- Information Dissemination and Sharing Layer: introduces multiple ways for information publication, such as: variable message signs, hotlines, traffic radios and the internet.

\section{Design of the Key Modules}

In this section the design of four key modules will be introduced, including: GIS and data-base, cubic information collection system, road network status analysis system, and multi-source information dissemination system.

\section{GIS and data-base}

Geographic Information System (GIS) is a basic module for the freeway traffic safety information platform. This system can express traffic resource and property information in the form of electronic map, and reproduce monitoring devices, traffic signs, publication infrastructure and so on in different graph layers which can be overlapped flexibly. The information shown in the graph can be updated in real-time. The map realizes functions for instance: browsing, zoom in and zoom out, positioning, and tagging.

The data-base behind GIS extends the massive data storage, and matches the GIS. Interfaces are provided for GIS to realize basic data-base operations, and for other cooperated data-bases to enable flexibility and interoperability.

\section{Cubic Information Collection}

To monitor and collect various information data, a cubic monitoring system is introduced. Several devices and sensors are used:

- Closed Circuit Television (CCTV): Remote and fixed focus cameras are used at pivotal sections, entrance and exit of the toll stations, tunnels and other service facilities.

- Mobile Video: Camcorders are carried on freeway cruisers and emergency service vehicles. They record traffic operation in real-time.

- Vehicle Global Position System (GPS): are installed on vehicles to get position information in the form of longitude and latitude in real-time. These data can be transferred to speed, OD, route and so on after processing.

- Meteorological Detectors: are placed along the freeway to collect the weather status. These data can also be obtained from the meteorological department.

- Internet of the Thing (IOT) based devices: are newly developed technologies taking advantages of the radio frequency identification devices (RFID). More elements can be identified and traced to get comprehensive dynamic and static data.

\section{Road Network Status Analysis System}

The road network status analysis is a core system to realize multi-view real-time analysis and traffic forecast. It efficiently supports the assistant decision for the freeway network operation management. Three subsystems are included: traffic status evaluation, traffic status forecast, and operation risk analysis.

The traffic status evaluation subsystem [3] is based on traffic data provided by the cubic information collection system. According to the management requirement from the monitoring center and branches, real-time quantitative assessments of congestion degree and road-network reliability are made for key sections. Congestion evaluation and operation reliability analysis [4,5] are two key modules for the subsystem. The congestion evaluation reflects workload on the freeway and the feeling of the road user. The operation reliability analysis utilizes probability to grade the operation status in certain service level. 
The traffic status forecast subsystem utilizes short-term traffic flow volume collected in real-time to deduce the operation status in the near future. The traffic control plan and contingency plan are supported by the forecast data. The detailed processing flow is shown in Fig. 2. By cooperating this subsystem with the previous traffic status evaluation subsystem, an overview for the freeway network traffic status can be given.

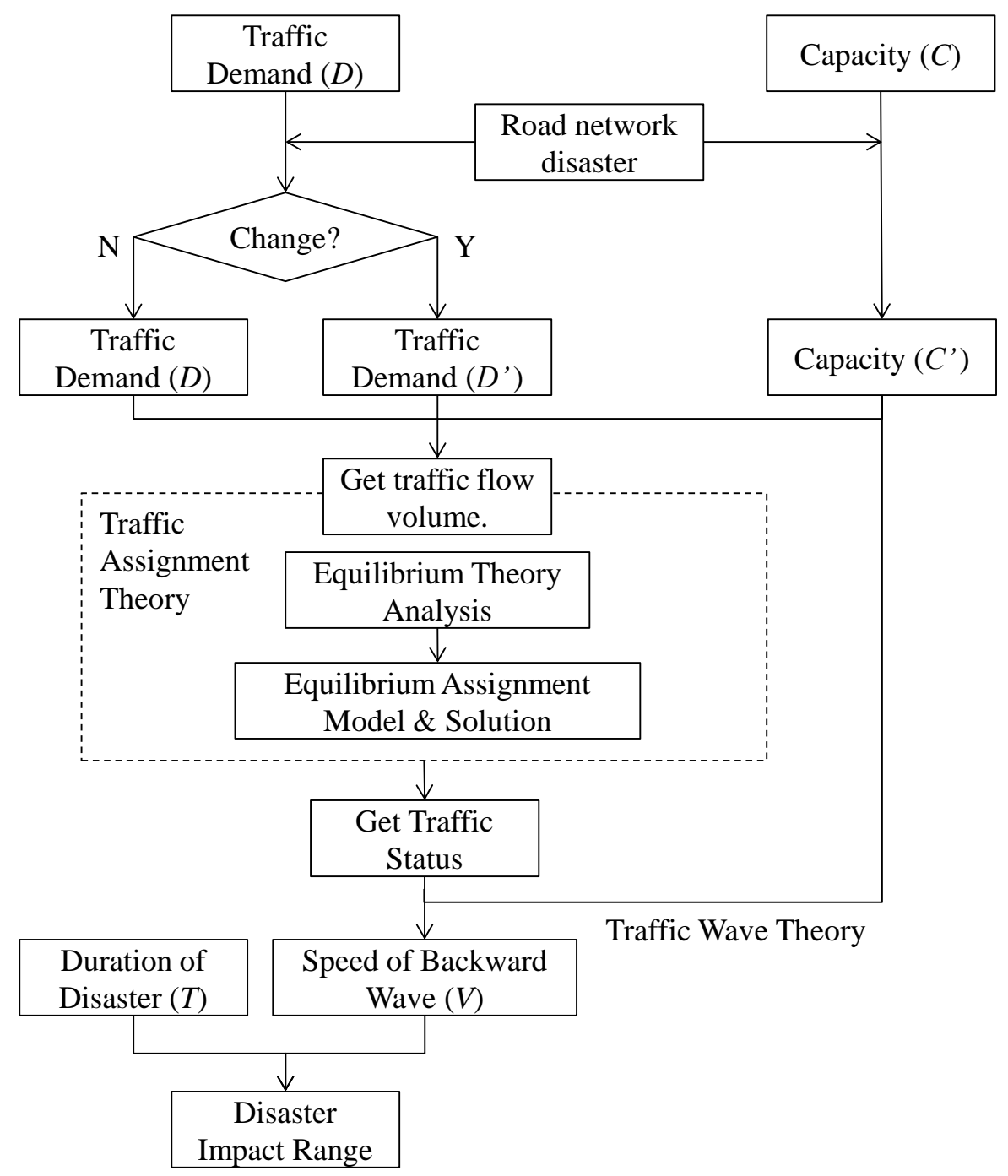

Fig. 2 Processing Flow of the Traffic Status Forecast

The operation risk analysis subsystem [6] evaluates risk for the freeway network operation according to the risk control level, and produces classified warning information. As shown in Fig. 3, the processing flow of the operation risk analysis follows three steps:

- Identify the freeway network operation risks.

- Setup the Key Performance Indicator (KPI) for risk evaluation.

- Make risk evaluation decisions and produce classified warning information. 

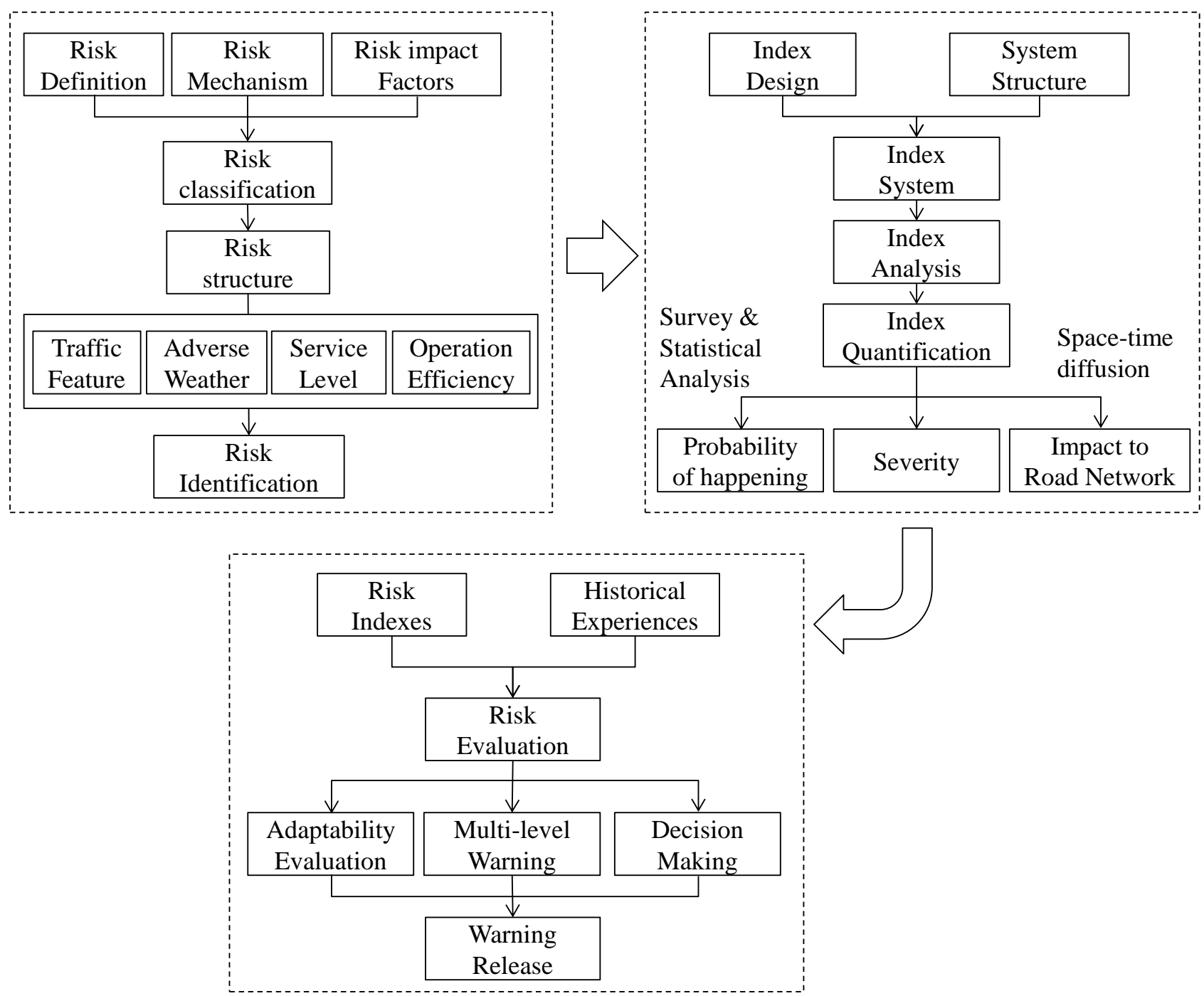

Fig. 3 Processing Flow of the Operation Risk Analysis

\section{Multi-source Information Dissemination System}

The multi-source information dissemination system can publish information, like intellegent control instuctions and assistent decisions, in various approaches to satisfy public transport demand and improve the service level. The provided functions include: variable message signs, hotlines, traffic radios, custom short message services (SMS) and the internet. The published data can be archived, so that operations like inquiring and exporting can be enabled.

\section{Conclusions}

With the rapid development of communication and information science, using the informational approach to improve the freeway traffic safety becomes popular. The information platform is proved to be an imperative part of freeway construction. Starting from the analysis of fuctional requirements, the freeway traffic safety information platform is proposed. Key modules are introduced with detailed design and analysis. The system and method proposed in this paper will be a reference for the freeway informatization construction in the future.

\section{Acknowledgement}

The authors acknowledge the supports of this paper by the National Natural Science Foundation of China \#51108214, the MOT Western Transportation Construction Science and Technology Program of China \#2009318223089, and the Key Traffic Science and Technology Project of Liaoning Province of China. 


\section{References}

[1] International Technology Scanning Program, Freeway Geometric Design for Active Traffic Management in Europe, Washington, Oct 2010.

[2] United States Government Accountability Office, SURFACE TRANSPORTATION: Efforts to Address Highway Congestion through Real-Time Traffic Information Systems Are Expanding but Face Implementation Challenges, GAO-10-121R, Washington, Nov. 2009.

[3] H. N. Liu. The Research of Highway Network Traffic Conditions Evaluation and Identification, Master Dissertation, Chongqing Jiaotong University, 2010.

[4] K. Chen. Models and Algorithms for Travel Time Reliability Assessment of Urban Road Networks Based-on Moving Source Data, Ph.D. Dissertation, Beijing Jiaotong University, 2008.

[5] D. H. Wang, H. S. Qi and C. Xu. Reviewing Traffic Reliability Research, Journal of Transportation Systems Engineering and Information Technology, vol. 5, 2010, pp. 12-21.

[6] T. Litman, S. Fitzroy. Safe Travels: Evaluating Mobility Management Traffic Safety Impacts, Victoria Transport Policy Institute, May. 2012. 